\title{
Nonequilibrium free energy of colloidal glasses under shear
}

\author{
M. T. Dang, ${ }^{1,2}$ R. Zargar, ${ }^{1}$ D. Bonn, ${ }^{1}$ A. Zaccone, ${ }^{3}$ and P. Schall ${ }^{1}$ \\ ${ }^{1}$ Van der Waals-Zeeman Institute, University of Amsterdam, The Netherlands \\ ${ }^{2}$ School of Education, Cantho University, Vietnam \\ ${ }^{3}$ Department of Chemical Engineering \& Biotechnology, University of Cambridge, U.K.
}

\begin{abstract}
The free energy of hard-sphere systems provides a direct link between the particle-scale structure and macroscopic thermodynamic properties. Here we employ this framework to investigate the shear-induced structure of a colloidal glass, and link it to its macroscopic mechanical and thermodynamic state. We measure the nonequilibrium free energy under shear from the free volumes of the particles, and monitor its evolution with the applied strain. Unlike crystals, for which the elastic energy increases quadratically with strain due to affine particle displacements, for glasses the free energy decreases due to non-affine displacements and dissipation, reflecting the ability of the glass to reach deeper free-energy minima. We model this decrease using the nonaffine shear modulus and a standard viscous dissipative term. Our model and measurements allow us to disentangle the complex contributions of affine and nonaffine particle displacements in the transient shear deformation of glasses.
\end{abstract}

\section{INTRODUCTION}

Unlike crystals that deform reversibly under small applied strain, amorphous materials such as glasses deform irreversibly even at vanishingly small deformations. This relaxation is a generic property of glasses and central to their mechanical properties, such as elasticity, timedependent anelasticity, and plastic flow, and its understanding remains a major challenge. This property of glasses is fundamentally connected to the lack of inversion symmetry: in most crystals, the lattice point symmetry guarantees local balance of forces by affine displacements, while in glasses, the amorphous short-range order precludes force balance by affine displacements alone, leading to additional non-affine motions to restore force balance locally under applied shear [1]. The usual description of glasses is also very different from crystals, involving a free-energy landscape picture in which the glasses seek to find ever lower free-energy minima by thermally activated hops over energy barriers. Such hops may also be shear-induced, causing accelerated aging due to the system being driven into deeper free-energy minima. These concepts have remained, however, rather abstract, because a direct connection between the shear-induced (non-affine) atomic displacements and the free energy is lacking for the simple reason that both are almost impossible to measure. Disentangling the complex contributions of affine and non-affine displacements and connecting them to the free-energy landscape of amorphous materials therefore remains a crucial challenge.

Colloidal glasses, on the other hand, do allow for a direct observation of single-particle dynamics, and consequently they have become much-studied model systems for the microscopic degrees of freedom of amorphous solids. Hard-sphere particles are known to undergo a colloidal glass transition at volume fraction $\phi_{g} \sim 58 \%$ [2]; since there are no interactions between the particles other than collisions, the free energy is uniquely defined by the entropy of the particles. Theory [3-6] and recent experiments $[7,8]$ have shown that the particle configurations then provide a direct route to the free energy of the system that is determined by geometry only. This opens up new opportunities to elucidate, experimentally, the relation between microscopic degrees of freedom and important bulk thermodynamic quantities of deformation.

In this paper, we experimentally study the shear deformation of glasses and connect the shear-induced microscopic structure to the macroscopic free energy of deformation. The main finding is that the free energy of the glass decreases with increasing deformation, whereas that of the crystal increases. To provide a quantitative analysis we then use a recent theoretical framework that generalizes Born's stability criterion for crystals to amorphous solids. In the model, strain-induced forces that balance by symmetry in centrosymmetric crystals, require additional non-affine displacements in amorphous solids to be relaxed. The framework we develop relates affine and non-affine displacements in amorphous solids, allowing us to link them to the elastic moduli, stress and free energy of the glass. The conclusion is that the free energy decrease is due to the non-affine displacements that allow the glass to find deeper free energy minima. This leads to a simple particle-scale picture of the transient deformation that is typical to glasses: the loss of interparticle contacts in the extensional sectors of the shear plane leads to loss of connectivity between the particles entailing a proliferation of non-affine displacements that enables the flow under the applied shear.

\section{EXPERIMENTS}

We use a colloidal glass consisting of sterically stabilized fluorescent polymethylmethacrylate (PMMA) particles with a diameter of $d=1.5 \mu \mathrm{m}$ and a polydisperity of $7 \%$, suspended in a density and refractive indexmatching mixture of cycloheptyl bromide and cis-decalin. A dense glassy suspension with particle volume fraction $\phi \sim 59 \%$, above the colloidal glass transition $\phi_{g}$ is prepared by diluting suspensions centrifuged to a sediment. 
(a)
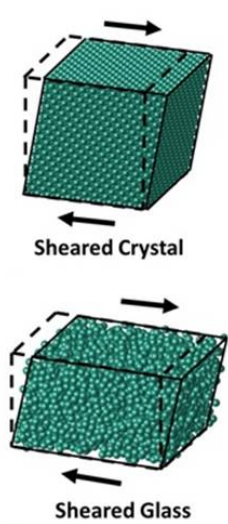

(b)
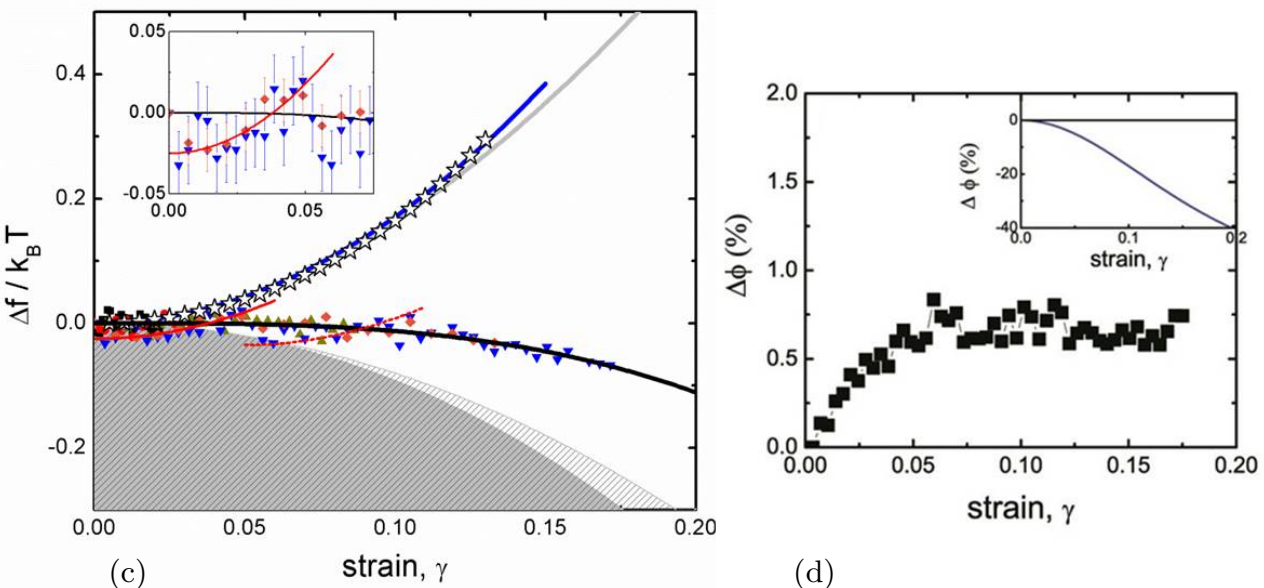

(d)

FIG. 1. (Color online) Changes in free energy and volume fraction upon deformation of crystals and glasses. Sketches of a sheared crystal (a) and glass (b) at strain $\gamma \simeq 0.18$. (c) Free energy per particle as a function of strain in two types of systems: a constructed fcc cystal displaying purely affine deformation (stars and up-curved blue parabolic fit) and experimental deformation of a colloidal glass at different shear rates (filled symbols and down-curved fit according to our model). Also indicated are the predicted affine (solid and dashed red upward curves) and non-affine components (light gray shaded area), and dissipation (dark gray shaded area). The affine component is given by $\Delta f(\gamma) / k_{B} T=18 e^{-0.9 \gamma} \gamma^{2}$ according to the first two terms of eq. (1). Symbols indicate shear rates of $\dot{\gamma}=1.5 \times 10^{-5} s^{-1}$ (black squares), $3 \times 10^{-5} s^{-1}$ (red circles), $6 \times 10^{-5} s^{-1}$ (olive triangles), $10^{-4} s^{-1}$ (blue triangles), $2.8 \times 10^{-4} s^{-1}$ (orange diamonds). Enlarged section of the free energy data in the inset (highest shear rates only) clearly shows the initial parabolic increase of the free energy at small strain, well fitted by the affine component only. A relaxation event leads to sudden free energy drop at a strain of $\sim 0.05$. The shear modulus corresponding to the parabolic increase is $\sim 34 k_{B} T / d^{3}$, roughly an order of magnitude smaller than shear moduli determineed by step-stress [17] or oscillatory measurements [18], possibly reflecting its frequency dependence. (d) Particle volume fraction change $\Delta \phi$ versus strain at shear rate $10^{-4} s^{-1}$ for the glass with initial volume fraction $\phi=0.59$. The inset shows the predicted volume fraction change versus strain in a simple expansion for marginal solids (see text).

At this high volume fraction, structural relaxations occur very slowly; at rest, we measure a glass relaxation time of $\tau=2 \times 10^{4} \mathrm{~s}$ by confocal microscopy as the time in which the particles' mean-square displacement becomes equal to their radius [9]. The suspension is loaded in a cell between two horizontal, parallel plates $65 \mu \mathrm{m}$ apart. A piezoelectric translation stage moves the top plate, applying shear at very slow rates between $\dot{\gamma}=1.5 \times 10^{-5} \mathrm{~s}^{-1}$ and $2.8 \times 10^{-4} s^{-1}$, of the order of the inverse relaxation time; these shear rates are significantly smaller than in other studies on colloidal flows [10-13]. The plates are immersed in a colloidal reservoir; hence the sheared suspension can dilate under constant osmotic pressure. We image the individual particles using confocal microscopy, and determine their positions in three dimensions with an accuracy of $0.03 \mu \mathrm{m}$ in the horizontal, and $0.05 \mu \mathrm{m}$ in the vertical direction [9]. This way, we follow the particle-scale structure of the glass under applied shear, from the undeformed state at strain $\gamma=0$ to $\gamma \sim 0.15$.

To link the structure to the thermodynamic properties of the sheared glass, we determine the free energy from the particle configurations obtained with confocal microscopy [7]. The central quantity of interest is then the free energy of deformation defined as $\Delta F \equiv F(\gamma)-F(0)$, i.e. the difference between the free energy $F(\gamma)$ of the deformed state under a strain $\gamma$, and the free energy
$F(0)$ of the unstrained glass at rest [14]. This quantity encodes the local affine and nonaffine distortions of the glass structure together with irreversible rearrangements due to relaxation. To determine it, we generate a Voronoi tessellation that divides the space into distinct, non-overlapping convex polyhedra. The free volume $v_{f i}$ associated with a Voronoi cell $i$ is then defined as the volume accessible to the center of mass of the particle, and is generated from the Voronoi cell by moving the faces normally inside over a distance of half the particle diameter. Note that this method is applicable to high densities [3], especially for glassy system [7] where the particles are densely packed. The total free energy of the hard-sphere system can then be expressed directly in terms of only the free volume as $F \simeq-k_{B} T \sum_{i=1}^{N} \ln \left(v_{f i} / \lambda^{3}\right)$, where $\lambda=\left(h^{2} / 2 \pi m k_{B} T\right)^{1 / 2}$ is the thermal wavelength [3, 7]. This allows us to track both reversible (equilibrium) [14] and irreversible (nonequilibrium) [15] contributions to the free energy of deformation.

\section{RESULTS AND DISCUSSION}

For a uniformly sheared, purely elastic crystal, the free energy should increase quadratically with applied strain, similar to the elastic deformation of a spring. 

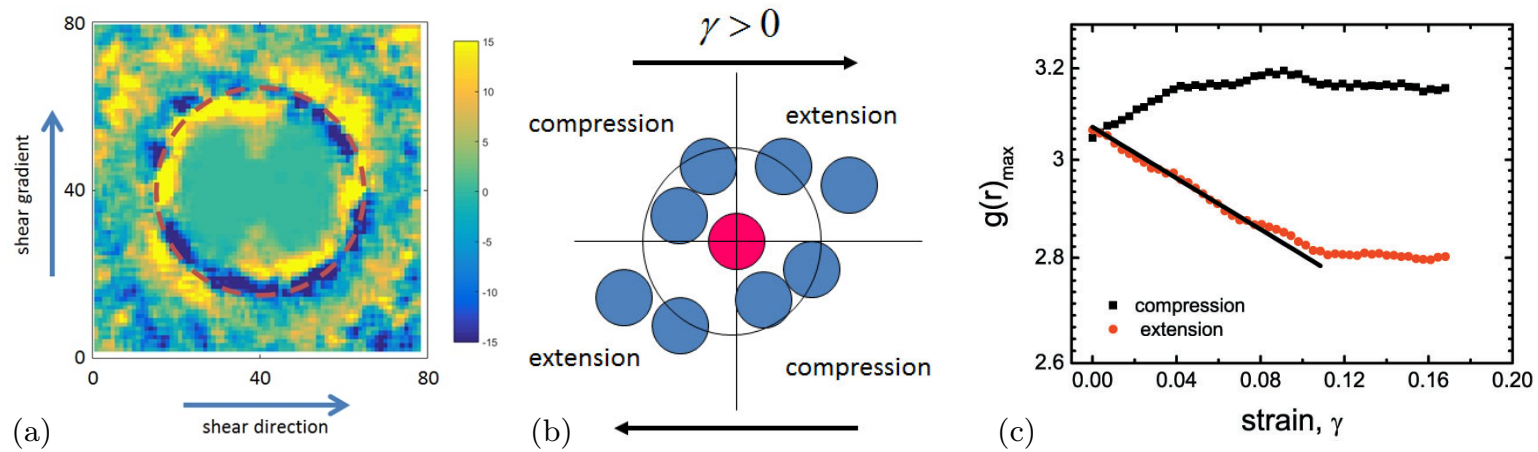

FIG. 2. (Color online) Shear-induced loss of structural connectivity. (a) Particle pair correlation function $g(r)$ in the shear plane. Color indicates the difference of $g(r)$ of the sheared glass $\left(\dot{\gamma}=10^{-4} s^{-1}\right)$ with respect to the undeformed state. (b) Schematic of the corresponding nearest-neighbor configuration under applied shear. The number of particles moving out of the cage in the extension direction is larger than that moving in along the compression direction, leading to net loss of connectivity. (c) Measured maximum of $g(r)$ in the compression (black squares) and extension (red circles) axis (plotted in logarithmic scale) as a function of strain (linear scale). Black fitting line has slope -0.9 .

This is indeed what we find when we construct an affine shear deformation on a computer-generated hard-sphere face-centered cubic (fcc) crystal, and use our method to compute the free energy (Fig. 1(c), open stars and blue fitting curve). Here, the particle volume fraction $\phi=54.5 \%$ was preserved using periodic boundary conditions. The curvature of the parabola indicates the elastic modulus, which we determine to be $\mu \sim 34.2 k_{B} T / d^{3}$, in good agreement with $\mu \sim 32.4 k_{B} T / d^{3}$ determined for $\phi \sim 54.3 \%$ in simulations [16]. In contrast, in the experiments, when we shear a colloidal glass, we measure a surprising decrease of the free energy (Fig. 1(c), colored closed symbols and black down-curved fit). This monotonic decrease indicates that microscopic degrees of freedom other than affine motions dominate the deformation with increasing strain.

Looking more closely, the data points suggest that the glass free energy in fact follows an upwards parabolic curvature initially (red solid curve). After this initial increase, at $\gamma \sim 0.05$, the free energy decreases abruptly, followed by another parabolic increase (red dashed curve), followed by another abrupt decrease, etc. These sudden drops suggest occurrence of significant relaxation events after some elastic loading. Such intermittency has been observed as important hallmark in the relaxation of stressed glasses, and we see it here even in the bulk free energy. This intermittency leads to an overall decreasing trend in the free energy, signaling significant relaxation of the glass under shear.

A possible mechanism decreasing the free energy is dilation; glasses can dilate under shear, increasing the free volume, and hence decreasing the free energy. Therefore we monitored, by particle counting, the volume fraction change upon deformation. However, we find that the changes are small; initially, the volume fraction even increases, indicating some compaction of the material, before it saturates at a constant value in the strain window where the free energy decreases, see Fig. 1(d). Clearly, dilation cannot account for the decrease in free energy. The small amount of compaction observed (Fig. 1(d)) can result from the osmotic pressure of the colloidal reservoir that is in direct particle exchange with the sheared colloidal layer. This compaction is in contrast to what is expected for sheared marginal elastic solids. We compute the dilation and resulting volume fraction change for sheared marginal elastic solids following [19], see Appendix. The resulting strain-dependent volume fraction $\phi(\gamma)$ decreases considerably with deformation (Fig. 1d, inset) to as much as $40 \%$ in the relevant range of strain values, in stark contrast to what we observe in our experiments on colloidal glasses (Fig. 1d).

Since dilation cannot explain the decrease in the free energy, we looked at other structural changes. We computed the pair correlation function $g(r)$ that indicates the probability of finding a particle at a distance $r$ with respect to the ideal gas distribution. Resolving $g(r)$ in the shear plane, we observe a significant distortion of the average nearest-neighbor structure under deformation, as shown in Fig. 2a, where we plot the difference of $g(r)$ between the deformed and undeformed states. Clearly, nearest neighbour distances become extended in the dilation direction and reduced in the compression direction, as expected for the shear geometry and consistent with earlier work [11, 20, 21]. At the same time, the angleaveraged nearest neighbor distance remains essentially unchanged as shown in the appendix (Fig. 6), confirming the overall constant density of the glass in agreement with Fig. 1d. As a result of the anisotropic distortion, particles become crowded along the compression direction, and less dense along the dilation direction, as schematically shown in Fig. 2b. This is reflected in the height of $g(r)$, which is a measure of the number of nearest neighbors. As shown in Fig. 2c, this height decreases significantly in the dilation direction, indicating neighbor loss, while it becomes only slightly larger along the compression direction, indicating only some neighbor gain. This imbal- 
ance results from the asymmetry of the hard-core repulsion rendering further approach of nearest neighbors prohibitively difficult. As the neighbor loss is not balanced by neighbor gain, this leads to an overall loss of neighbours and to destabilization. The resulting imbalance of forces causes the particles to move non-affinely to restore the local force balance $[1,22]$. Because these nonaffine displacements perform internal work against the potential of mean force (the potential-field of our "lattice"), this process results in an overall negative contribution to the free energy [23-25]. We note that by looking in the shear plane, we have focused here on the most obvious nonaffine contributions; non-affine rearrangements may exist in other planes as well (see e.g. recent work on a sheared emulsion in [26]), but are typically less dominant, as shown by the three-dimensional nature of shear rearrangements in colloidal glasses [27].

To obtain a quantitative description of the free energy of deformation, we first consider the affine part, $F_{A}=\frac{1}{2} G_{A} \gamma^{2}$, where the shear modulus $G_{A}=\frac{2}{5 \pi} \frac{\kappa \phi}{\sigma} n_{b}$ in the linear regime, according to the Born-Huang theory of lattice dynamics [28]. Here, $n_{b}$ is the number of nearest neighbors and $\kappa$ the spring constant associated with a nearest-neighbor bond. In our hard-sphere glass, the "bonds" between neighbors arise from the entropic attraction (akin to depletion attraction) [29] that relates the first peak of the radial distribution function $g(r)$ to an attractive minimum in the pair potential of mean force $V_{\text {eff }} / k T=-\ln g(r)$. This also defines the effective elastic spring constant as $\kappa=\left[d^{2} V_{\text {eff }} / d r^{2}\right]_{r=\sigma}$ between two bonded neighbors. The number of bonded neighbors is given by the integral of the first peak of $g(r)$, which yields $n_{b}^{0} \approx 12$ for the static hard-sphere glass [30]. Under applied shear, this value decreases as the loss of neighbors in the dilation direction is not balanced by the particle gain in the compression direction $[20,32]$. We model this loss of neighbors by assuming that the local cage dynamics is governed by the Smoluchowski equation with shear [33]. The general spherically averaged solution is $g(r, \gamma)=\exp \left[-V_{\text {eff }} / k T+h(r) \gamma\right]$ with $h(r)<0$, which after integration translates to $n_{b}(\gamma)=n_{b}^{0} \exp [-A \gamma]$, corresponding to a decrease of the height $g_{c}\left(r_{\max }\right) \propto \exp (-A \gamma)$ of the first peak of $g(r)$ in the extension direction. This estimate is in excellent agreement with the exponential dependence shown in Fig. 2c; the numerical factor $A$ follows from a fit to the data, and we obtain $A=0.9$ [34].

The reduced connectivity leads to growing non-affine contributions decreasing the shear modulus. Assuming that the rigidity of the material breaks down at the isostatic limit $n_{b}=6$ [23], the total shear modulus $\left(G_{A}-G_{N A}\right) \propto\left(n_{b}-6\right)$, meaning that $G_{N A}=\frac{2}{5 \pi} \frac{\kappa \phi}{\sigma} 6$. The total reversible free energy then follows from integration: $F_{e l}(\gamma)=\frac{1}{5 \pi} \frac{\kappa \phi}{\sigma}\left[n_{b}(\gamma)-6\right] \gamma^{2}$. This total elastic part of the free energy, with fitting parameters specified below, indeed fits the initial parabolic increase of the free energy as shown by the red curve in Fig. 1c.

To account for the relaxation-related decrease of the

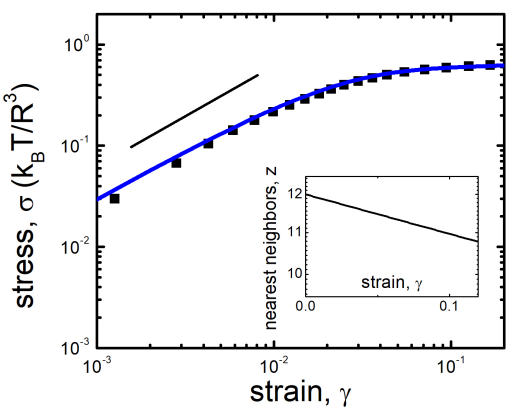

FIG. 3. (Color online) Comparison of model prediction (blue line) and experimental data (black squares) of stress $\sigma$ as a function of strain $\gamma$ (log-log scale). To measure the stress reliably at these small shear rates, we used smaller particles with diameter $d_{1}=50 \mathrm{~nm}$, resulting in shear stresses larger by a factor $\left(d / d_{1}\right)^{3} \sim 3 \times 10^{4}$. All data were measured at the same volume fraction $\phi=59 \%$ and similar Peclet number. The black line has slope 1 to guide the eye. Inset: theoretical prediction of the average number of nearest neighbors (log scale) as a function of strain (linear scale).

free energy, we also include a term related to irreversible rearrangements. We model these irreversible changes here by a standard continuum dissipative term. In standard dissipation, the dissipated energy associated with continuously ramping the strain at strain rate $\dot{\gamma}$ is $W_{\text {diss }}=\int_{0}^{t} \sigma(s) \dot{\gamma} d s$, where the stress $\sigma(t)=$ $\dot{\gamma} \int_{0}^{t} G(s) d s$ [35] with $G(t)$ the (strain dependent) relaxation modulus. Using a standard viscoelastic model, the relaxation modulus has the general form $G(t)=$ $G+G_{R} \exp [-t / \tau]^{\beta}$, where $\tau=\eta / G_{R}$ is the global relaxation time, $\eta$ the viscosity [36] and $G_{R}=G_{0}-G$, with $G_{0}$ the instantaneous (infinite-frequency) shear modulus. For the standard linear viscoelastic solid [35], one has $\beta=1$, while for many glassy materials the relaxation is stretched-exponential with $\beta<1$. Thus, inserting the stretched-exponential expression for $G(t)$ in the integral, and using $t=\gamma / \dot{\gamma}$, one obtains $W_{\text {diss }}$ as a function of $\dot{\gamma}, \gamma$ and $G_{R}$. Focusing on the limit of very low $\dot{\gamma}$ as in our experiment, the leading term in a Taylor expansion around $\dot{\gamma}=0$ is $W_{\text {diss }} \approx \frac{1}{2} G_{R} \gamma^{2}$, independent of $\beta$.

The total nonequilibrium free energy of deformation then is $F(\gamma)=F_{0}+F_{e l}(\gamma)-W_{\text {diss }}(\gamma)$, where $F_{0}$ is the strain-independent, "ground-state" energy of the metastable minimum (inherent structure) of the glass, $F_{e l}=\frac{1}{2} G \gamma^{2}$, with $G=G_{A}-G_{N A}$ the elastic (reversible) energy containing both affine and non-affine contributions, and $W_{\text {diss }}(\gamma)$ the dissipated energy due to irreversible rearrangements. Using the terms derived above, the final expression for the free energy of deformation is

$$
F(\gamma)=F_{0}+\frac{1}{5 \pi} \frac{\kappa \phi}{\sigma}\left[n_{b}^{0} \exp (-A \gamma)-6\right] \gamma^{2}-\frac{1}{2} G_{R} \gamma^{2}
$$

To test our model quantitatively, we simultaneously fit the free energy data in Fig. 1c using Eq.(1) (black line) 
and stress-strain curves of a colloidal glass measured independently with a rheometer [13], see Fig. 3. Here, the stress is computed by adding the elastic and viscous contributions: $\sigma=\sigma_{e l}+\sigma_{v i s}$, where $\sigma_{e l}=\partial F_{e l} / \partial \gamma$, and the standard viscous expression $\sigma_{v i s}=\eta \dot{\gamma}\left(1-e^{-\gamma / \dot{\gamma} \tau}\right)$. We obtain excellent simultaneous fits up to large strains, validating our model for the nonequilibrium free energy with elastic and viscous components. The calculated number of nearest neighbors (Fig. 3, inset) decreases with strain, reflecting the loss of connectivity and the propagation of non-affine contributions. This decrease of nearest neighbors saturates eventually as shown in the experimental $g(r)$ for strains $\gtrsim 0.1$, see Fig. 2 c.

In summary, we connect the microscopic structure of sheared glasses to their nonequilibrium free energy of deformation by combining colloidal shear experiments with generalized stability theory of amorphous materials. Nonaffine contributions decrease the shear modulus and thereby the initial elastic (parabolic) rise of the free energy, while dissipation - modeled here by a standard continuum dissipative term - explains the overall decrease of the non-equilibrium free energy with strain. The applied shear thus allows the glass to explore lower free energy states by forcing structural rearrangements that would otherwise be frozen or take much longer time by thermal aging alone. The measured decrease of the free energy under deformation is indeed of the order of, albeit a bit smaller than the free energy decrease of $0.1-0.2 k_{B} T$ obtained upon aging the glass for a few days. This decrease should saturate at larger strain $(\gamma \gg 0.1)$, when the glass acquires a steady-state structure under the applied constant shear rate. Our results thus provide insight into the role of non-affine displacements and dissipation in the transient deformation of glasses [14]. While our hardsphere colloidal glass allows direct measurement of the free energy and underlying microscopic distortions, the proposed mechanism should apply to molecular glasses as well. For those molecular glasses, the strong hardcore repulsion is replaced by a steep repulsive potential $\left(\propto r^{12}\right)$ leading to similar shear-induced loss of connectivity and growth of non-affine dynamics.

\section{APPENDIX}

\section{A. Dilatancy of marginal solids}

To estimate the dilatancy of a marginal solid, following [19], we compute the dilatant strain in a quasistatic response of an isotropic elastic solid according to

$$
\epsilon=\frac{1}{2} R_{p} \gamma^{2}
$$

where $R_{p}$ is the Reynolds coefficient. This Reynolds coefficient is defined as

$$
R_{p}=\left(\frac{\partial G}{\partial p}\right)_{\gamma}-\frac{G}{E}=\left(\frac{\partial G}{\partial \phi}\right)_{\gamma}\left(\frac{\partial \phi}{\partial p}\right)_{\gamma}-\frac{G}{E},
$$

where $E=2 G(1+\nu)$ is the Young's modulus, $G$ the shear modulus and $\nu=\frac{1}{3}$ the Poisson ratio. Thus, the free energy can be expressed as

$$
\frac{F}{V}=\frac{F_{0}}{V}+\frac{1}{2} G \gamma^{2}-p \frac{1}{2} R_{p} \gamma^{2} .
$$

The volume fraction of the dilated system follows as

$$
\phi=\phi_{\gamma=0}(1+\epsilon)^{-3} .
$$

For hard-sphere crystals, the equation of state is, within the framework of cell theory [37, 38]

$$
\frac{p}{\rho k_{B} T}=\frac{3}{1-\phi / \phi_{c p}}
$$

where $\phi_{c p}=0.74, \rho=\phi / V_{0}$, and $V_{0}$ is the particle volume. Thus,

$$
\frac{\partial p}{\partial \phi}=\frac{3 k_{B} T}{V_{0}} \frac{1}{\left(1-\phi / \phi_{c p}\right)^{2}} .
$$

Values of the shear modulus as a function of volume fraction can be obtained from Ref. [16]. $G=1.189 \times$ $10^{6} \phi^{19.37}+39.23\left(k_{B} T / \sigma^{3}\right)$. By interpolating this data to the volume fraction of our computer generated crystal, $\phi=54.5 \%$, we obtain $G=48.5 k_{B} T / d^{3}$ and $E=129$ $k_{B} T / d^{3}$, where the hard-sphere diameter $d=1.5 \mu \mathrm{m}$. It then follows that the Reynolds coefficient

$$
R_{p}=\left(\frac{d G}{d P}\right)_{\gamma}-\frac{G}{E}=\left(\frac{\partial G}{\partial \phi}\right)_{\gamma}\left(\frac{\partial \phi}{\partial p}\right)_{\gamma}-\frac{G}{E}=3.63 .
$$

The resulting change of volume fraction with respect to this dilatancy $R_{p}$ is shown in Fig. $4 \mathrm{a}$, and the corresponding free energy change is shown in Fig. $4 \mathrm{~b}$.

(a)
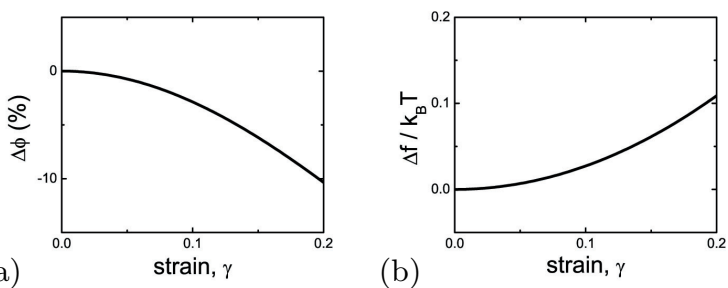

FIG. 4. Volume fraction and resulting free energy change of sheared crystal as a function of shear strain in the linear approximation.

For hard-sphere glasses, the equation of state is [39]

$$
\begin{aligned}
& p=-\frac{k_{B} T}{V_{0}} \phi^{2} \frac{d}{d \phi} \ln \left({\frac{\phi_{\max }}{\phi}}^{1 / 3}-1\right)^{3} \\
& =\frac{k_{B} T}{V_{0}} \phi^{2} \frac{\phi_{\max }}{\phi\left(\phi_{\max }-\phi\left(\frac{\phi_{\max }}{\phi}\right)^{2 / 3}\right)},
\end{aligned}
$$


where $\phi_{\max }=0.64$.

Thus, we determine that

$$
\frac{d p}{d \phi}=\frac{k_{B} T \phi_{\max }}{3 V_{0}} \frac{3\left(\frac{\phi_{\max }}{\phi}\right)^{1 / 3}-2}{\phi\left(\frac{\phi_{\max }}{\phi}\right)^{2 / 3}-2 \phi_{\max }+\phi_{\max }\left(\frac{\phi_{\max }}{\phi}\right)^{1 / 3}} .
$$

The shear modulus as a function of volume fraction of our hard-sphere glass can be obtained from Ref. [38]. By interpolation, we determine that for our volume fraction, $\phi=59 \%, G \sim 960 k_{B} T / d^{3}$ and $E=2.55 \times 10^{3} k_{B} T / d^{3}$. With these values, we obtain $R_{p} \sim 24$. The resulting change of volume fraction is shown in Fig. 5a, and the corresponding free energy change is shown in Fig. 5b.

(a)

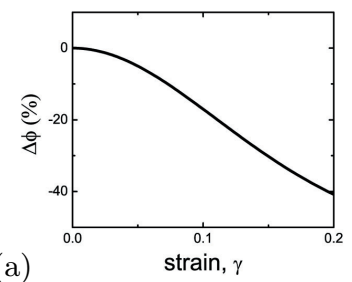

FIG. 5. Volume fraction and resulting free energy change as a function of strain for a sheared glass with linear dilatancy.

\section{B. Experimental evidence of negligible dilatancy in the glass}

In the main text, we investigate the dilatancy in the sheared glass by monitoring the volume fraction by particle counting. Here, we present an alternative way to check for dilation based on the angle-averaged pair correlation function that indicates the probability of two particles to be separated by a distance $r$. We estimate the dilation in the deformed state from the minimum of the mean-square difference $\Delta^{2}$ between the $g(r)$ curves of the deformed and undeformed glass as a function of a linear stretching $\alpha$ that transforms $r$ to $r^{\prime}=r \alpha$. We show $\Delta^{2}$ as a function of $\alpha$ in Fig. 6, inset; its minima are at $\alpha \sim 1$, all very close to 1 , indicating neither compression nor dilation. Close evaluation of the minima $\alpha_{\text {min }}$ for all deformed states in the interested strain window confirms this, indicating maybe a small compression of less than one per mill in deformed states compared to the quiescent state (Fig. 6, main panel). Similarly to the manuscript, these closely constant values contrast with the strong dilatancy predicted by the linear theory, and indicate substantial nonlinear behavior, even at small strain.

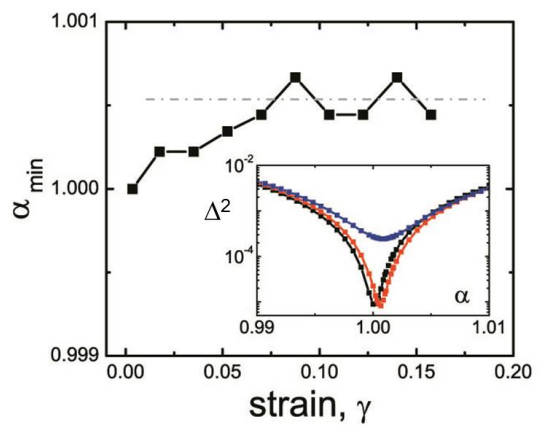

FIG. 6. (Color online) Dilation parameter $\alpha_{\text {min }}$ versus shear deformation determined from the minimum of the meansquare difference of $g(r)$ of the deformed and undeformed glass (inset). Dash-dotted line is a guide to the eye.

\section{ACKNOWLEDGMENTS}

This work is funded by the Netherlands Organization for Scientific Research (NWO). P. S. acknowledges support by a Vici fellowship from NWO. We thank D. Frenkel, E. Lerner, G. Petekidis, and M. Hoffmann for useful discussions.
[1] A. Zaccone, Mod. Phys. Lett. B 27, 1330002 (2013); A. Zaccone and E.M. Terentjev, Phys. Rev. Lett (2013).

[2] G. Biroli and J.P. Garrahan, J. Chem. Phys. 138, 12A301 (2013).

[3] T. Aste and A. Coniglio, Europhys. Lett. 67, 165 (2004).

[4] J.E. Lennard-Jones and A.F. Devonshire, Proc. Roy. Soc., 163A, 53 (1937).

[5] R.J. Buehler, et al., J. Chem. Phys. 19, 61 (1951).

[6] R.J. Speedy, J. Chem. Soc. Faraday Trans. 276, 693 (1980).

[7] R. Zargar, B. Nienhuis, P. Schall, and D. Bonn, Phys. Rev. Lett. 110, 258301 (2013).

[8] R. P. A. Dullens, D. G. A. L. Aarts, W. K. Kegel, Proc. Natl. Acad. Sci. 103, 529 (2006).
[9] V. Chikkadi, G. Wegdam, D. Bonn, B. Nienhuis and P.Schall, Phys. Rev. Lett. 107, 198303 (2011).

[10] R. Besseling, L. Isa, P. Ballesta, G. Petekidis, M. E. Cates, and W. C. K. Poon, Phys. Rev. Lett. 105, 268301 (2010).

[11] X. Cheng, J. H. McCoy, J. N. Israelachvili, I. Cohen, Science 333, 1276 (2011).

[12] F. Westermeier, D. Pennicard, H. Hirsemann, U. H. Wagner, C. Rau, H. Graafsma, P. Schall, M. P. Lettinga and B. Struth, Soft Matter 12, 171 (2016).

[13] D. Denisov, M. T. Dang, B. Struth, G. Wegdam and P. Schall, Sci. Rep. 3, 1631 (2013).

[14] L. D. Landau and E. M. Lifshitz. Course of Theoretical Physics, Volume VII: Theory of Elasticity. ButterworthHeinemann, 3rd edition, (1980). 
[15] W. Ebeling, I. M. Sokolov, "Statistical Thermodynamics and Stochastic Theory of Nonequilibrium Systems", World Scientific, Singapore (2005).

[16] S. Pronk and D. Frenkel, Phys. Rev. Lett. 25, 90 (2003).

[17] G. Petekidis, D. Vlassopoulos and P. N. Pusey, J. Phys.: Condens. Matter 16, S3955 (2004).

[18] K. van der Vaart, Y. Rahmani, R. Zargar, D. Bonn and P. Schall, J. Rheol. 57, 1195 (2013).

[19] B. P. Tighe, Granular Matter 16, 203-208 (2014).

[20] J. Zausch and J. Horbach, Europhys. Lett. 88, 60001 (2009).

[21] N. Koumakis, M. Laurati, S. U. Egelhaaf, J. F. Brady, and G. Petekidis, Phys. Rev. Lett. 108, 098303 (2012).

[22] A. Zaccone and E. Scossa-Romano, Phys. Rev. B (2011); A. Zaccone, J.Blundell, E.M. Terentjev Phys. Rev. B (2011).

[23] S. Alexander, Phys. Rep. 296, 65 (1998).

[24] J.F. Lutsko, J. Appl. Phys. 65, 8 (1988)

[25] A. Lemaitre and C. Maloney, J. Stat. Phys. 123, 2 (2006)

[26] V. V. Vasisht, S. K. Dutta, E. Del Gado, and D. L. Blair, Phys. Rev. Lett. 120, 018001 (2018).

[27] P. Schall, D. A. Weitz and F. Spaepen, Science 318, 1895 (2007).

[28] M. Born and H. Huang, Dynamical Theory of Crystal Lattices, Oxford University Press 1954

[29] Hansen and MacDonald, Theory of Simple Liquids, Academic Press 2005.
[30] Unlike in the liquid, a good part of the bonded nearest neighbors in the glass are long-lived, and contribute to the long-time elasticity of the glass [31].

[31] F. Puosi and D. Leporini, J. Chem. Phys. 136, 041104 (2012).

[32] V. Chikkadi, S. Mandal, B. Nienhuis, D. Raabe, F. Varnik and P. Schall, Europhys. Lett. 100, 56001 (2012).

[33] J. K. G. Dhont, An Introduction to Dynamics of Colloids (Elsevier, Amsterdam, 1996).

[34] This shear-induced cage breakdown may be even bigger than what we can infer here on the basis of the static $g(r)$. The static $g(r)$ includes also those neighbors which fluctuate very fast over long distances, and hence do not contribute to stress-transmission and elasticity. The decrease of the number of neighbors as derived from the behavior of $g(r)$ is therefore probably an underestimation of the real decrease, which might be stronger if one could separate the contributions of slow (permanent) and fast (non-permanent) neighbors.

[35] C. Zener, Elasticity and Anelasticity of Metals (Chicago Univ. Press, Chicago, 1965).

[36] K. Binder and W. Kob, Glassy Materials and Disordered Solids (World Scientific, Singapore, 2011).

[37] M. A. Rutgers et al., Phys. Rev. B 53, 5043 (1996).

[38] R. Zargar, Thermodynamics and Vibrational Modes of Hard Sphere Colloidal Systems, PhD thesis (2014).

[39] R. D. Kamien and A. J. Liu, Phys. Rev. Lett. 99, 155501, (2007). 